\title{
Utility-based Analytical Model for representing user QoE
}

\author{
Manzoor Ahmed Khan ${ }^{1}$, Jie Lu ${ }^{2}$, and Muhammad Amir Mehmood ${ }^{3}$ \\ ${ }^{1}$ DAI Labor, Technical University Berlin, Germany \\ manzoor-ahmed.khan@dai-labor.de \\ ${ }^{2}$ Technical University, Berlin, Germany \\ ${ }^{3}$ Deutsche Telekom Laboratories, Technical University, Berlin, Germany
}

\begin{abstract}
Next Generation Mobile Networks (NGMNs) together with smart phones evolution have opened new opportunities to deliver mobile services, including voice, data and multimedia applications to the users. However this conflation has brought several challenges in terms of Quality of Experience - $Q o E$ of users. Mainly due to degradations with timevarying characteristics in the presense of link heterogeneity, varying packet loss and codec switching. In this paper we propose an analytical quality prediction model which predicts user QoE for conditions that are typically occurring in NGNMs such as network handovers, codec switching and different packet loss rates due to adverse wireless networking conditions. We augment our model with service price as another key factor in QoE representation. We validate quality prediction of our model with subjective tests. Our results show that the quality prediction by our model has correlation of $r=0.923$ with subjective ratings.
\end{abstract}

Keywords: Utility function, NGMN, QoE, VoIP

\section{Introduction}

Wireless internet access has grown significantly over the last decade from, a barely sufficient bandwidth provided by GRPS and EDGE, to the medium sized UMTS/HSDPA, UMTS/HSPA+, WiFi, WiMax and towards the high speed envisaged by LTE. Also smart phones such as iphone and Android are equipped with multiple wireless network interfaces e.g. 3G UMTS or WiFi. This has given users an opportunity to be always connected to the Internet anywhere and anytime. Moreover, it has provided users a choice to select the best network according to their own preference driven by their network choice, quality acceptance and cost effectiveness [1].

While bandwidth offered by these technologies is increasing, the link layer properties are also exhibiting time-varying characteristics. These factors may force users to react according to the network conditions. For instance, due to adverse nature of wireless medium it is imperative to change speech bandwidth inorder to maintain a certain quality of service. This action sometimes results in changing of speech codec during the ongoing call i.e., codec changeover and/or change of network point of attachment i.e., network handover. The study of the these new conditions and modeling the impact on speech quality prediction for NGMN is the key focus of this work.

On one hand, the availability of these high-speed access technologies have encouraged the users to use Mobile VoIP while they are on the move, on the other hand the physical layer implementation of these wireless technologies have different impact on real time applications. For instance, HSDPA link exhibits more fluctuations than WiFi due to shared downlink. The primary reasons for this is base station scheduling which allocate resources only to the users having good wireless link conditions. WiFi is also interference limited technology and link transmission is greatly dependent on the simultaneous demand of the wireless channel resources. However WiFi as compared to HSDPA performs better in terms of throughput and latency. These differences in access technologies are the key factor of different quality of experience of users for different services. Due to this fact, network service providers which are offering superior quality VoIP services by employing wideband codecs sampled at $16 \mathrm{kHz}$ in their network require service adaptation to narrowband codec at $8 \mathrm{kHz}$ when facing adverse network conditions. 
The usage of voice services over IP networks has grown significantly. Skype for instance dominates the market with its over 521 Million accounts and over 40 million active users that generate most amount of international traffic. Mobile VoIP is also becoming popular and it is being incorporated into the mobile phones using SIP protocol. This protocol allows exchange of SIP messages into the device and then RTP may be used to manage voice path. Mobile VoIP demands high-speed wireless communications such as WiFi, WiMax or HSDPA. Also these access networks open opportunities to deliver new services to the mobile users.

Mobile service adoption is clearly dependent on quality received by the users as users expect same quality of fixed phone while on roaming. Thus call quality monitoring is really crucial for NGMN service providers. The call quality monitoring may be based on network transmission metrics, but also on the evaluation of the transmitted audio signal arriving at the user end terminal. This information can be further mapped into the quality experienced by the end user. Real user perception measurement requires time-consuming and expensive subjective tests but also provide the ground truth and most accurate Mean Opinion Score (MOS), which express the mean quality score of a group of users according to ITU-T Rec. P.800 [2]. The test complexity limits the applicability of such a quality measurement. These limitations form the basis and serve as the driving force for an alternate approach of instrumental quality prediction.

The functional principle of quality prediction models can rely on evaluation of transmission parameters measured in networking layer e.g. E-model [3], or on the analysis of the signal waveform measured at the output of the transmission system e.g. Perceptual evaluation of speech quality - PESQ [4]. These approach allow to derive the quality index without performing subjective tests and is based on either parametric approach or signal-based approach. However, these models have been tested for only certain conditions related to fixed networks and their applicability for NGMN typical networking conditions are still limited. This underscore the stark need of another modeling approach which can capture NGMN effects along with external factors such as service price. Our model is premised on the fact that along with typical conditions in NGMN's e.g., network handovers, codec switchovers and packet loss, service price offered by a particular provider plays a major role in mobility management based on QoE.

In this paper, we present analytical quality prediction model based on the utility function of a user, that predicts quality for NGMN typical conditions.Our main contributions are as follows:

- Analytical representation of user satisfaction using utility function specifically concentrating on impact of handover and codec switchover on the user satisfaction for heterogeneous services.

- Validating the estimated satisfaction values attained from the proposed utility function for different scenarios (by comparing the estimated satisfaction values of utility function to the values attained from the experimental work).

We structure rest of the paper as follows. In Section 2, we discuss related work, next in Section 3 we present user satisfaction function. We continue with discussion on satisfaction criteria in Section 4. In Section 5 we show our experimental methodology and we compare experimental and model results in Section 6. We conclude the paper in Section 7.

\section{Related work}

Speech quality assessment has been the subject of many researchers [5]. Various different models and their modifications have been proposed for signal based speech quality prediction model such as PESQ and WB-PESQ [6][7]. Similarly many efforts have been made in parametric based model such as ITU-T E-model [3] and its various extensions. In the context of speech quality assessment when network handovers and codec switchover are prevalent, a comprehensive quality assessment study has been carried out by Möller et al. [8], which encompasses the changes in the user quality of experience for various different roaming scenarios. Their study highlight the fact that packet loss is the most dominant factor in NGMN conditions and network handover (only) 
has minimal impact compared to packet loss and codec switching. Another interesting fact from that study reveals that when packet loss is high, changing codecs does not impact quality much. However, when the packet loss is low, changing codec has high impact on the quality.

In another recent study by Mehmood et al. [9] authors show that WB-PESQ model failed to predict quality under some NGMN conditions. It was shown with experimental evidence that WB-PESQ underestimates quality due to (i) wideband-narrowband speech codec switching, (ii) speech signal fading during codec switching, and (iii) talk-spurt internal time-shifting due to jitter buffer instabilities. This highlights the fact that current signal based models are still not tuned for NGMN typical conditions and needs enhancement. Similar studies of NGMN conditions carried by Blazej et.al. [10] pointed out that the parametric model such as E-model was unable to predict the quality where narrowband and wideband codecs are present in a single call. Their suggested codec-switching impairment factor to E-model improved the correlation of the E-model prediction to auditory tests to $r=0.937$. Chen et.al. [11] have tried to find out user satisfaction index of voip user, however their work is limited to Skype user satisfaction only.

Utility theory, basically a concept from economics has been greatly used in wireless communication. [12,13] modeled network selection using utility functions, Das et al. [14] helps users take decision over service price by modeling users' utility that captures data delay considerations. A number of utility models with numerous technical / non-technical parameters such as QoS degree, service pricing, security, power consumption, throughput/bandwidth, delay, etc. are existent in the literature. The utility modeling and selection of parameters are basically driven by the scalability and their application to a specific problem. Expecting users as the center of all services in future wireless landscape, utility theory is a natural candidate that can be used to translate user satisfaction. Utility function answers the basic question, how much does a user value the service? Utility, when defined as the function that captures user satisfaction for communication service, user keeps on consuming services as long as the user's satisfaction is met [15]. The failure of signal-based and parametric-based models has prompted the need of an alternate model which could cover NGMN typical conditions and provide accurate prediction as compared to the existing models. Therefore in this paper, we endeavor to bridge this gap by taking advantage of analytical tools and present an analytical model. However, to the best of our knowledge, there are no current analytical quality models to estimate quality level fluctuations as a consequence of time-varying conditions such as network handovers and codec switchovers which are typical conditions in NGMN environments.

\section{Why is QoS QoE mapping needed?}

The arguments justifying the QoS $\sim$ QoE mapping also serve to be the motivation force for this work. We now briefly list few of the arguments as follows; i) Owing to the short term contractual agreements of users with operators, the objective of network operators will be driven by the satisfied user pool. To attain this objective, network operators will be interested in knowing the user behavior towards service in different context. This provisions analytical function that can estimate user satisfaction for different services for online optimization. Having estimated the user QoE for any service, an operator is in better position to select the optimal strategy(ies) to increase its objective function both in competitive and cooperative market settings.

\section{User Satisfaction Function}

In this section, we present the primer on utility function and real-time applications followed by the proposed utility function.

\subsection{Background on Utility Function}

The concept of utility function is defined by the notation " $\succeq$ ", which comes from the field of Economics. It is an abstract concept and is derived largely from Von Neumann and Morgenstern(1947). Utility function is designed to measure the user's satisfaction, which is subjective in 
nature. A satisfaction function measures users' relative preference for different levels of considered parameter values. For instance, given a number of items characterized by various parameters, a user decision of selecting any of these items will be based on relative values item specific parameters. Thus preference relation can be defined by the function, $U: X \rightarrow \Re$, that represents the preference $\succeq$ for all $x$ and $y \in X$, if and only if $U(x) \geq U(y)$. Here the concept of marginal satisfaction is worth mentioning, that represents the additional satisfaction gained from each extra unit of attribute. This concept is basic to demand theory which states that marginal satisfaction diminishes as the consumption of an item increases. Basically a satisfaction function should satisfy non-station and risk aversion properties [16].

Definition 1 Let $u\left(\rho_{i}\right)$ represent the satisfaction function for attribute $i$, from the attribute space $\Sigma=\left\{\rho_{1}, \ldots, \rho_{m}\right\}$, then non-station property states that satisfaction $u\left(\rho_{i}\right)$ increases with attribute value (given that attribute value has the expectancy the greater the better) i.e. $u^{\prime}\left(\rho_{i}\right)>0$.

Definition 2 Risk-aversion property states that satisfaction function is concave meaning thereby marginal satisfaction decreases with increasing value of attribute (provided that attribute value has the expectancy the greater the better), i.e. $u^{\prime \prime}\left(\rho_{i}\right)<0$.

The proposed satisfaction function can capture the user-preferences over multiple attributes including those having major influence on user QoE. In the broader sense, these attributes may be categorized in three different expectancies, i) the larger the better, ii) the smaller the better, and iii) the nominal the better. The combined effect of these attributes dictate the user satisfaction level.

\subsection{Background On Real-time Applications}

Streaming and conversational traffic classes can be combined in real-time applications, which are commonly termed as inelastic or rigid applications. Examples of real-time symmetric applications include teleconferencing, Videophony and VoIP. Generally real-time applications are constrained by minimum amount of bandwidth i.e., application is admitted only when the demand for minimum required bandwidth is met. Such stringent requirement on bandwidth are typically represented by a step function, which means that as soon as the demand for application required bandwidth is met, users' maximum satisfaction is reached, and for a slight reduction in this bandwidth below the minimum required bandwidth, users satisfaction is zero. In our case, bandwidth offers may be defined by different codecs. However, bandwidth is not the only factor that affects the satisfaction of user, factors like packet loss, delay, handover, and codec switchover costs are important parameters to be considered specifically for mobile users.

\subsection{Environment Assumptions}

Heterogeneous applications have differing preferences over QoS parameters, such as delay, packet loss, and jitter etc. Values of these parameters can in turn be translated into consumer satisfaction. In this research work, we focus on real-time applications i.e., voice application. Basically as all other services real-time voice application can also be defined in terms of delay and packet loss rate, and its sensitivity to these parameters pose service quality requirements. We term application preferences as minimum application required QoS, whereas user preferences are represented by user-profile. Thus the lower bound on user preferences is set by the minimum application required QoS. We assume that different user profiles define user types. Such user distinction is an attractive solution of adaptive applications, however for real-time applications we assume that all users are equally treated. We further assume that delay impact is negligible, this assumption is strengthened by the fact that we are considering non-interactive speech, and also the assumption is in line with E-model, where below a certain delay threshold $(<177 \mathrm{~ms})$ non-interactive communication is not affected. However, we assume that packet loss rate deteriorates the user satisfaction, as it varies in time and such time varying conditions are the consequence of varying wireless channel conditions, which in turn is influenced by the interference caused by users in the cell and other medium 
characteristics. We also assume that user experiences vertical handovers and codec switch overs during the life time of a call. We consider network technologies of different characteristics, let these be represented by the set $W$, that contains as elements the broadband and cellular network technologies. It is also assumed that handover execution delay is within permissible bounds, however we do capture its impact on the user satisfaction.

\subsection{Utility Function Representation of User Satisfaction}

Table 1: Notations and their description

\begin{tabular}{ll}
\hline Notation & Description \\
\hline \hline$u_{j}()$. & Utility function of user $j$ \\
$k$ & Index of service type \\
$c$ & The class of service (VoIP in this case) \\
$c o$ & Index of codec \\
$w$ & Index of network technology \\
$\pi$ & the price user pays against the service \\
$n_{o, k, c, c o, w}$ & Number of users $(k, c, c o, w)$ with operator $o$ \\
$b$ & Bandwidth \\
$p l$ & Packet loss \\
$\sigma$ & Measures the rate of decay of user's satisfaction. \\
$\zeta$ & Handover costs. \\
$r_{0}$ & Index of operator reputation \\
$s_{o}$ & Index of operator offered security \\
$\varphi$ & Codec-switchover costs respectively. \\
\hline
\end{tabular}

In this section, we take into consideration the handover costs and codec switching costs in the formulation of the utility function. The proposed user utility function (satisfaction function) is the function of packet loss, handover, codec switchover costs but also reputation and security and reputation indexes of the operator. We summarize in Table-1 the notations and their description used in the paper. Let $u_{j}($.$) be the utility function of user j$, and is given by

$$
\begin{aligned}
& u_{j, k, o, c, c o, w}(p l, \psi \zeta, \varphi)=\tilde{u}_{o}\left(\frac{b_{k, o, c}}{n_{o, k, c}}\right) \mu_{\bar{w}, k, o, c}^{\bar{c} o} e^{-\sigma\left(p l_{\bar{w}, k, o, c}^{\bar{c} o}+\psi\left(\frac{b_{k, o, c}}{n_{o, k, c}}\right) \zeta_{w}^{\bar{w}}\right)} \\
& -\psi\left(\frac{b_{k, o, c}}{n_{o, k, c}}\right) \tilde{\mu}_{w \longrightarrow \bar{w}}^{c o} e^{-p l_{w, k, o, c}^{c o} \varphi_{c o}^{\overline{c o}}}-\pi_{k, o, c, c o, w}-f\left(r_{o}, s_{o}\right)
\end{aligned}
$$

where $\mu_{\bar{w}, k, o, c}^{\bar{c} o}$ represents the maximum achievable MOS value in the network technology $w \in$ $W=\{W L A N, H S D P A\}$ using codec $c o \in\{G 722, G 722.2, G 711\}$ for operator $o$, pl represents the packet loss rate. Here $\psi$ takes the value of 1 , when the call handsover from high bandwidth to lower bandwidth technology or from wideband codec to narrowband codec, and -1 otherwise. $\tilde{\mu}_{w \longrightarrow \bar{w}}^{c o}$ represents the constant gain or loss of MOS value when codec switch over takes place and their values are dictated by the network and codec characteristics. The function $\pi$ represents the pricing function to the users. The term $\tilde{u}_{j, o}\left(\frac{b_{o, k, c}}{n_{o, k}}\right)$ is a function of network state $n=\left(n_{o, k, c o, w}\right)_{o, k, c, c o, w}$ and the offered bandwidth $b_{o, k, c}$ by the operator $o$. The collection $n$ is the vector that represent the total number of users those request the service of specific class under specific codec and handover. The function $f$ is a generic function that takes into consideration the reputation and the security of the operator (an increasing function in each of the component).

It is noted that $\mu_{\bar{w}, k, o, c}^{\bar{c} o} \neq \mu_{w, k, o, c}^{c o}$, where $(c o, w) \neq(\overline{c o}, \bar{w})$, it shows that users quality rating is dependent on the networking conditions e.g., WiFi with stable network bandwidth along with a superior quality wideband codec sampled at $16 \mathrm{kHz}$ provides best results, whereas HSDPA with 
its fluctuating bandwidth due base station based scheduling and high link layer retransmissions is expected to provide lesser quality even if superior quality codecs are used [8]. Value of $\zeta$ is dictated by the handover type i.e., $\zeta_{b m}>\zeta_{m b}$, where $b m$, and $m b$ represents the break before make, and make before break approaches respectively. Generally the value of $\sigma$ is dictated by the application type and the underlying network technology.

Since the proposed utility function is monotonically decreasing in all the considered parameters i.e., with increase in a considered parameter value the user gets more irritated. We therefore, introduce the term cost value that represent the aggregated effect of different parameter values. Let $L$ represents the set of considered parameters, then $L=\{p l, \zeta, \varphi\}$. We define the cost value as: $\mathcal{K}=\sum_{z_{m} \in L} z_{m}$, where $z_{m}$ is the individual cost contribution of parameter $m$. Thus the function can now be written as:

$$
u_{j}(\mathcal{K}, p l, \psi \zeta, \varphi)=\left\{\begin{array}{lr}
0 & \text { if } \quad \mathcal{K} \geq \mathcal{K}_{\text {max }} \\
\mu_{w}^{c o}+\psi \tilde{\mu}_{w}^{\bar{c} o} & \text { if } \quad \mathcal{K}<\mathcal{K}_{a c} \\
\mu_{w}^{c o} e^{-\sigma(\mathcal{K}-p l \varphi)}-\psi \tilde{\mu}_{w}^{c o} e^{\mathcal{K}}-(p l+\psi \zeta) \quad \text { if } \quad \mathcal{K}_{a c}<\mathcal{K}<\mathcal{K}_{\max }
\end{array}\right.
$$

where $\mu_{w}^{c o}+\psi \tilde{\mu}_{w}^{\overline{c o}}$ represents the maximum achievable utility depending on the user context and users association with a technology and codec for the voice call, $\mathcal{K}_{\max }$ is the maximum tolerable cost by the user $j$, it can also be interpreted as cost threshold, above which user is no more satisfied. $\mathcal{K}_{a c}$ represents the acceptable cost of the user, and it is taken as the ideal cost threshold for maximum satisfaction of user.

Consider the $u_{j}(\mathcal{K})$ of user $j$, where $\mathcal{K}$ is the aggregated cost computed from the technical characteristics of operators' offers. This dictates that there always exists an upper bound for the considered parameters due to technological constraints or user preferences / requirements. Such bounds define different regions of utilities as shown in Equation-2. Where the two obvious regions are translated as fully satisfied and fully irritated regions bounded by $\mathcal{K}<\mathcal{K}_{a c}$ and $\mathcal{K} \geq \mathcal{K}_{\text {max }}$ respectively. Third region bounded by $\mathcal{K}_{a c}<\mathcal{K}<\mathcal{K}_{\max }$ shows the satisfaction behavior of user following the function given in Equation 2. All the three regions define the user satisfaction of users. Let us scale the user satisfaction on the same lines as the ITU standard for QoE measurements. We scale the satisfaction function between $0 \sim 5$, where 5 represents the maximum and 0 minimum user satisfaction, i.e. $u_{j}(\mathcal{K}) \in[0,5]$.

The region of proposed utility function represented by $\mu_{w}^{c} e^{-\sigma(\mathcal{K}+p l \varphi)}-\psi \tilde{\mu}_{w}^{\tilde{c}} e^{\mathcal{K}-(p l+\psi \zeta)}$ should be twice differentiable on interval $\left[\mathcal{K}_{a c}, \mathcal{K}_{\text {max }}\right]$. Fulfilling this requirement guarantees that the user satisfaction level does not change drastically with a small change in the $\mathcal{K}$ value and marginal satisfaction is regular. Similarly, increase in value of considered parameter, say packet loss ratio above certain threshold results in near zero user satisfaction, therefore user behaves indifferently for parameters values above some threshold value. This implies the convexity of the satisfaction curve.

In addition the utility function should also satisfy the following requirements:

$$
\begin{array}{cr}
u_{j}(\mathcal{K})=0 & \forall \mathcal{K} \geq \mathcal{K}_{\text {max }} \\
u_{j}(\mathcal{K})=\mu_{\text {max }} & \forall \mathcal{K}<\mathcal{K}_{a c}
\end{array}
$$

where $\mu_{\max }$ represents the maximum user utility.

\subsection{User Equilibrium Characterization}

We denote the second term in Equation 1 by $\nu_{j}$, which is expressed as function of $p l, \psi, \zeta, \varphi$ and the type, the class, the codec and the handover. We assume that the performance index $p l$ 
decreases with the number of users $n_{o, k, c}$. We propose the following form:

$$
\begin{aligned}
\nu_{j, o, k, c, c o, w}(p l, \psi, \zeta, \varphi)= & \mu_{\bar{w}, k, o, c}^{\bar{c} o} e^{-\sigma\left(p l_{\bar{w}, k, o, c}^{\bar{c} o}+\psi\left(\frac{b_{k, o, c}}{n_{o, k, c}}\right) \zeta_{w}^{\bar{w}}\right)} \\
& -\psi\left(\frac{b_{k, o, c}}{n_{o, k, c}}\right) \tilde{\mu}_{w \longrightarrow \bar{c}}^{c o} e^{-p l_{w, k, o, c}^{c o} \varphi_{c o}^{\bar{c} o}}
\end{aligned}
$$

Now, we formulate the user problem based on the utility functions. Each user $j$ optimizes $u_{j}$ depending on its codec, handover, type, class of services, and operator. By fixing the operators offer (prices, technology, network availability etc), we analyze the interaction between the users in an autonomous and self-optimizing manner. We use the notion of equilibrium (Cournot, Nash, correlated, sequential, hierarchy etc.) which characterizes the outcomes. Here we restrict our attention to the packet loss $p l$ component but the delay function can be incorporated as well.

1. The equilibria in absence of handover: In this case, we omit the index $w$. A pure equilibrium is a configuration of choices of users, the total number of user profiles with specific codec, type, class represented by $\left(n_{o, k, c, c o}\right)_{o, k, c, c o}$ such that if user $j$ moves to $(\bar{o}, \overline{c o})$

$$
u_{j, o, k, c, c o}\left(n_{o, k, c, c o}\right) \geq u_{j, \bar{o}, k, c, \overline{c o}}\left(n_{\bar{o}, k, c, \overline{c o}}^{\prime}\right)
$$

where $n_{o, k, c, c o}^{\prime}=n_{o, k, c, c o}-1$ if user $j$ has moved from $(o, c o), n_{o, k, c, c o}+1$ if user moves to $(o, c o)$ and equal to $n_{o, k, c, c o}$ if user does not move.

2. The equilibria in absence of codec switchover: In this case, we omit the index co. A pure equilibrium is a configuration of choices of users, the total number of user profiles with specific handover, type, class represented by $\left(n_{o, k, c, w}\right)_{o, k, c, w}$ such that if user $j$ moves to $(\bar{o}, w)$

$$
u_{j, o, k, c, w}\left(n_{o, k, c, w}\right) \geq u_{j, \bar{o}, k, c, \bar{w}}\left(n_{\bar{o}, k, c, \bar{w}}^{\prime}\right)
$$

where $n_{o, k, c, w}^{\prime}=n_{o, k, c, w}-1$ if user $j$ has moved from $(o, w), n_{o, k, c, w}+1$ if user moves to $(o, w)$ and equal to $n_{o, k, c, c o, w}$ if no moves.

3. The equilibria in presence of both handover and codec switchover:

In this case, we take on both indexes $w, c o$. A pure equilibrium is a configuration of choices of users, the total number of user profiles with specific codec, type, class represented by $\left(n_{o, k, c, c o, w}\right)_{o, k, c, c o, w}$ such that if user $j$ moves to $(\bar{o}, \overline{c o}, \bar{w})$

$$
u_{j, o, k, c, c o, w}\left(n_{o, k, c, c o, w}\right) \geq u_{j, \bar{o}, k, c, \overline{c o}, \bar{w}}\left(n_{\bar{o}, k, c, \overline{c o}, \bar{w}}^{\prime}\right)
$$

where $n_{o, k, c, c o, w}^{\prime}=n_{o, k, c, c o, w}-1$ if user $j$ has moved from $(o, c o, w), n_{o, k, c, c o, w}+1$ if user moves to $(o, c o, w)$ and equal to $n_{o, k, c, c o, w}$ if no move.

For the mixed equilibria of the selection problem with strict support are characterized by the indifference conditions. This means that one needs the solve the system $u_{j, o, k, c, c o, w}\left(x^{*}\right)=$ $\max _{\bar{o}, \overline{c o}, \bar{w}} u_{j, \bar{o}, k, c, \bar{c}, \bar{w}}\left(x^{*}\right)$ where $x^{*}$ the vector with component $x_{o, k, c, c o, w}^{*}$ of probabilities of being in the configuration $(o, k, c, c o, w)$.

In real world scenarios user satisfaction is influenced by different technical and non-technical parameters, however non-technical parameters are out of the scope of this paper. Concentrating further on technical parameters, the effect of these parameters can either be dependent on each other or independent of each other. In the scope of this research work, we consider the parameters that are mutually independent in terms of its impact on the users satisfaction. We model the utility function so that it captures the codec switch over handover costs independently, and their independent impact is added to the degradation caused by the packet loss costs. Such utility function modeling can be understood following lemma-1.

Lemma 1 The proposed satisfaction function is invariant under various independent parameters. 
Proof. Assume that user $i^{\prime} s$ satisfaction is the function of packet loss only. Let it be represented by $U_{i}(p l)$, and is given by $U(p l)=e^{-\sigma(p l)}$, then for any constant parameter $x$ it is straight forward to prove $U(x+p l)=e^{-\sigma(p l+x)}$, which results in $U(x+p l)=U(p l) e^{-\sigma(x)}$.

Remark 1 Having known the expected MOS value of a user in different user contexts, a decision maker is provided with suitable and necessary information for policy making, taking different quality improvement measures, and adopting certain service price offering strategies that in turn increases the operators' payoffs. Given the utility function the proportion of MOS degradation by different parameters can also be estimated.

Remark 1 is true for both the scenarios, i) where individual MOS effecting parameters are considered, ii) combined impact of various MOS effect parameters are considered. However when it comes to the later case, the proposed satisfaction function can be generalized for a number of dependent and independent parameters by introducing the relevant functions of newly introduced parameters and associating it to the satisfaction function using additive sum concept. In this case associating weights to parameters is intuitive, which takes into account the sensitivity of the parameter.

Remark 2 The satisfaction function can be replaced by an equivalent satisfaction function for

$$
u(y)=A u(y)+B
$$

where $A>0$ and $B$ is arbitrary. Hence it is possible to standardize the satisfaction function for a particular point $\varsigma$.

Example - 1 Let the the standardization of satisfaction function is required for $\varsigma=0$, the for the satisfaction (simplified form of equation-1).

$$
u(y)=\mu \cdot e^{-\sigma(y)}
$$

It can be noted that for $y \rightarrow \infty$, the satisfaction is bounded and tends to the finite value zero.

\section{Satisfaction Criteria}

In this section, we investigate the performance of proposed utility function for single and multiple criteria.

\subsection{Single attribute utility function}

In a single criterion utility function, the utility function captures user satisfaction for the possible degradation effect introduced by the criterian. Consider an ideal scenario, where a static user experiences zero delay, no vertical handover and no codec switchovers. In such a case users satisfaction is fully dependent on the packet loss rate.

$$
u_{i}(p l):=\mu_{w}^{c o} e^{-\sigma \cdot p l} \quad \forall p l \in\left[p l_{a c}, p l_{\max }\right]
$$

Let us consider that user the is associated to a specific codec say $c o=G 711$, and with network technology $w=H S D P A$, here $\mu_{w}^{c o}$ represents the maximum achieveable MOS value in this scenario, which is achievable for packet loss less than $p l_{a c}$. We plot the MOS curve for this scenario in Fig 1a. In addition we also investigate single attribute utility function for a different network technology $w=W L A N$, and broadband codec $G 722$, and plot the user satisfaction curve for this scenario as well 1a. As can be observed that $\mu_{W L A N}^{G 722}>\mu_{H S D P A}^{G 711}$, this strengthens the argument presented in section 3. One more point to be noticed here is that $\sigma$-value in WLAN is greater than in HSDPA, which results in greater decay of MOS value with increasing packet loss. The proposed utility function behavior is validated for various scenarios in later sections; i.e., keeping the mentioned setting for experiments we obtain values for different values of packet loss $(0 \%, 10 \%, 20 \%)$. 


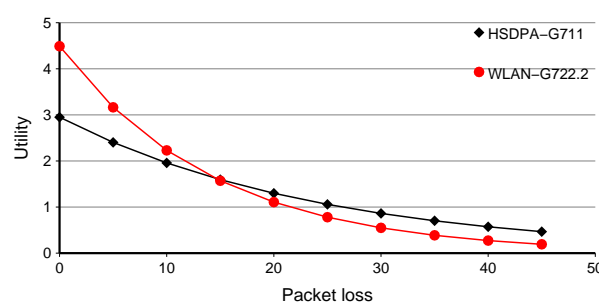

(a) Plot of utility values considering single at-(b) tribute (packet loss)

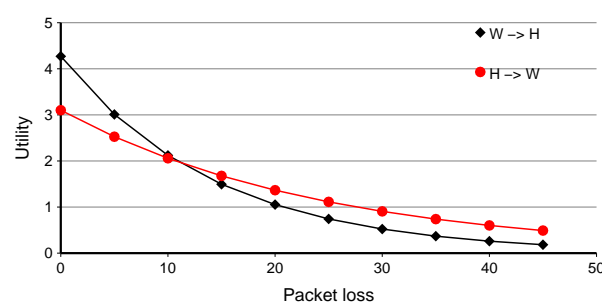

(b) Plot of utility values considering multiple attributes (packet loss \& handover cost)

Fig. 1: Impact of various attributes on user satisfaction

\subsection{Multiple attributes utility function}

In a multiple attribute utility function, the utility function captures user satisfaction for the possible degradation effect introduced by the combined effect of multiple criteria. Now we consider a scenario, where a user experiences vertical handovers, and his satisfaction is influenced by both packet loss and handover costs, which is represented by:

$$
u_{i}(p l):=\mu_{w}^{c o} e^{-\sigma \cdot(p l+\psi \zeta)} \quad \forall p l \in\left[p l_{a c}, p l_{\max }\right]
$$

Let us consider a specific scenario, where a user is initially associated with network technology WLAN and codec $G 722.2$. The maximum achieveable MOS value for this user is $\mu_{W L A N}^{G 722.2}$, now that user experiences a vertical handover to $H S D P A$, he experiences a further degradation (this degradation is represented by $\psi \zeta$, in this case $\psi=-1$ ). We plot the MOS curve for this scenario as shown in Fig 1b.
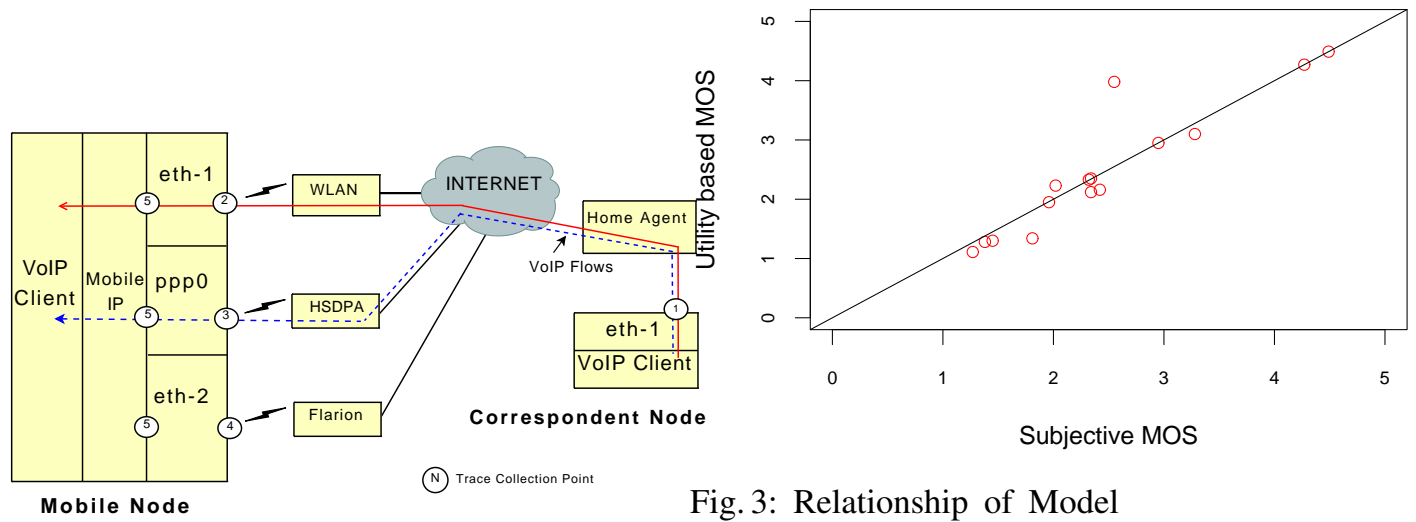

Fig. 2: Mobisense experimental setup for VoIP services in NGMNs

Fig. 3: Relationship of Model quality estimation and user quality ratings (correlation $r=$ 0.923).

The plot resembles exactly the one shown in Fig 1a for different value of packet loss, but shifted by a small value representing the handover cost. In the similar fashion, we plot MOS curve for users initially associated to HSDPA network technology and handsover to WLAN network technology, such handover introduces a gain to users satisfaction (therefore $\psi=1$ ). Equation 9 captures user satisfaction for both mentioned scenarios for multiple attribute utility function. In 
general Fig $1 \mathrm{~b}$ presents somewhat similar behavior as in Fig 4 shifted by the cost of handover cost in either direction depending on the value of $\psi$.

\section{Experiments}

In this section, we discuss our experimental methodology for carrying out the controlled experiments and then conduct auditory tests for NGMN conditions.

\subsection{Mobisense Testbed}

In order to perform quality of experience study, we rely on Mobisense testbed which was designed to investigate user perceived quality in NGMN using VoIP as the primary application. The testbed depicted in Figure 2 allows mobile clients to perform network handovers and voice codec changeover while keeping on-going calls. The testbed uses Mobile IPv4 as a solution to enable seamless handovers between different radio access technologies [17]. Thus, the main network elements of the testbed are the Mobile Node $(\mathrm{MN})$, the Corresponding node $(\mathrm{CN})$ and the Mobile IPv4 Home Agent (HA). The setup includes various different access technologies such as WLAN/WiFi, UMTS/HSDPA and Flash-OFDM (pre-WiMax release) that are used to emulate an integrated NGMN environment.

The CN and MN were deployed on laptops with linux 2.6.18.2 and HA was configured on a Cisco 2620XM router with Cisco IOS 12.2(18r). Based on SIP protocol we choose P JPROJECT [18] framework as voip client to play speech files. We employed seamless codec switching scheme by Wältermann et al. [19]. The detailed description of design and the capabilities of Mobisense testbed are outlined in [20].

\subsection{Methodology}

We pre-selected NGMN conditions with the combination of (i) Network handovers between different technologies, (ii) Codec-switching between narrowband codec and wideband codec, and (iii) Different packet loss rates. We use ITU-T G.711 log PCM codec at $64 \mathrm{~kb} / \mathrm{s}$ for narrowband (NB) with native packet loss concealment and ITU-T G.722.2 (AMR-WB) codec at $23.05 \mathrm{~kb} / \mathrm{s}$ for wideband transmission. For benchmarking purposes we select few conditions with a single network, a single codec and $0 \%$ packet loss. We establish the quality bounds by using these benchmark conditions. Of course one could argue why not to test all combinations but carrying out a full factorial combination of subjective tests is practically infeasible. We use netem packet shaper for degrading VoIP packets as shown in Figure 2.

All calls were initiated at $\mathrm{CN}$ and terminated at MN. During on-going calls network conditions were imposed depending upon the requirements of network handovers or codec switching. At MN, speech samples were collected and processed for subjected tests according to ITU-T P.800 [2]. Further we select speech samples for both male and female speakers to obtain a balance set of speech samples. These samples were then presented to 24 subjects for quality assessment. For the quality rating, 5-point Mean Opinion Score (MOS) scale was used. More details of these tests can be found in [8]. The overall assessment along with conditions is listed in Table 2.

\section{Results and Model Validation}

In this section, we compare subjective ratings with our model predictions. In principle the model should be able to predict estimations for most of the conditions. We first check correlation of the predicted values of our model with the subjective test ratings obtained in Table 2. The results are shown in Figure 3. The concentration of most of the points around the diagonal line is an indicator of a good match between model predicted values and the actual ground truth obtained from 


\begin{tabular}{|c|c|c|c|c|c|c|}
\hline No. & Network & Codec & Ppl & Subj. & Model & Diff. \\
\hline 1 & $\mathrm{~W} \rightarrow \mathrm{H}$ & 722.2 & 0 & 4.27 & 4.27 & 0.00 \\
2 & $\mathrm{~W}$ & 722.2 & 0 & 4.49 & 4.49 & 0.00 \\
3 & $\mathrm{~W} \rightarrow \mathrm{H}(\mathrm{b})$. & $722.2 \rightarrow 711$ & 0 & 2.34 & 2.35 & -0.01 \\
4 & $\mathrm{H} \rightarrow \mathrm{W}(\mathrm{a})$. & $711 \rightarrow 722.2$ & 0 & 2.55 & 3.98 & -1.43 \\
5 & $\mathrm{H} \rightarrow \mathrm{W}$ & 711 & 10 & 2.42 & 2.158 & 0.26 \\
6 & $\mathrm{~W}$ & 722.2 & 10 & 2.02 & 2.23 & -0.21 \\
7 & $\mathrm{H} \rightarrow \mathrm{W}(\mathrm{b})$. & $711 \rightarrow 722.2$ & 20 & 1.81 & 1.339 & 0.47 \\
8 & $\mathrm{~W} \rightarrow \mathrm{H}(\mathrm{a})$. & $722.2 \rightarrow 711$ & 0 & 2.32 & 2.33 & -0.01 \\
9 & $\mathrm{~W} \rightarrow \mathrm{H}$ & 722.2 & 10 & 2.34 & 2.12 & 0.22 \\
10 & $\mathrm{H}$ & 711 & 0 & 2.95 & 2.95 & 0.00 \\
11 & $\mathrm{H} \rightarrow \mathrm{W}$ & 711 & 0 & 3.28 & 3.10 & 0.18 \\
12 & $\mathrm{H}$ & 711 & 10 & 1.96 & 1.95 & 0.01 \\
13 & $\mathrm{~W} \rightarrow \mathrm{H}(\mathrm{b})$. & $722.2 \rightarrow 711$ & 20 & 1.38 & 1.28 & 0.1 \\
14 & $\mathrm{~W}$ & 722.2 & 20 & 1.27 & 1.11 & 0.16 \\
15 & $\mathrm{H}$ & 711 & 20 & 1.45 & 1.3 & 0.15 \\
\hline
\end{tabular}

Table 2: Comparison of Analytical Model estimates and Auditory Judgments (MOS).

Ppl: Packet loss in \%; Diff.: Difference of subjective MOS \& Utility-based Model MOS; W: WLAN; H: HSDPA; $\rightarrow$ : Handover/Changeover, a./b. : Changeover before/after handover.

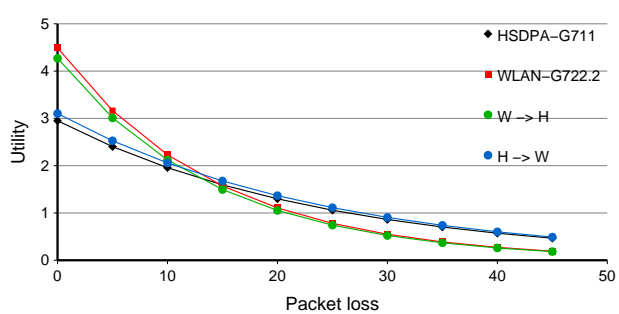

Fig. 4: Plots of Utility for single and multi-attribute scenarios

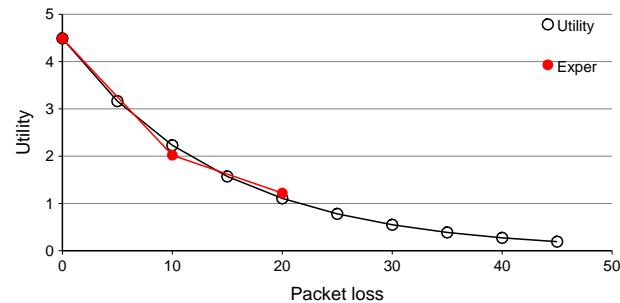

Fig. 5: User associated with WLAN \& G722.2(No Handover/codec switchover

the subjects. There is a correlation of 0.923 between model predicted values and the subjective ratings.

We note that for the conditions \# 1, 2, 3, 810 and 12 in Table 2, our model perfectly match the subjective ratings. For conditions \#5, 9, 11, 14 and 15 model is off by maximum of 0.26 MOS value. However, inspite the fact that we see a high correlation we have certain conditions e.g., condition \#4 and 7 where our model deviates from the subjective ratings. We plot different curves for both the utility function and experimental data in different scenarios so that behavior of utility function for different parameters can be investigated. In Figure-5, we consider the scenario where the user is associated to WLAN and G722.2, in this scenario we study the impact of packet loss rate. As evident from the Figure-5 proposed utility function almost overlaps the experimental results for low packet loss values, very small deviation is observed, when packet loss rate approaches $20 \%$. Although experimental results are not available for greater packet loss than $20 \%$, the proposed model can confidently estimate the behavior for higher packet loss values. A number of similar curves were obtained for scenarios like i) user associated to HSDPA and G711 with experiencing no handover and codec switch over, ii) user associated to WLAN, G722.2 and experiencing handover to HSDPA, iii) user associated with HSDPA and handsover to WLAN, iv) Codec switchover, v) Simultaneous codec switchover and network handover etc. In all the mentioned scenario user utility function was validated against the experimental results. 


\section{Summary and Future Work}

The emergence of new access technologies along with evolution of smart phones have paved a way of any time anywhere connectivity for mobile users. However this new paradigm has brought along many challenges for network service providers. The biggest of them is to maintain a certain level of quality for multimedia streams of their customers.

With this context, in this paper we propose a utility-based analytical quality prediction model which predicts user QoE for conditions that are typical to NGNMs such as network handovers, codec switching and different packet loss rates due to adverse wireless networking conditions. We validate quality prediction of our model with subjective tests. Our results show that the quality prediction by our model has correlation of $r=0.923$ with subjective ratings.

In future, we plan to include more NGMN typical conditions and to extend our model for new services e.g. IPTV, video conferencing and video telephony.

\section{References}

1. I. Ganchev, G. Morabito, R. Narcisi, N. Passas, S. Paskalis, V. Friderikos, A. S. Jahan, E. Tsontsis, C. Bader, J. Rotrou, and H. Chaouchi. Always best connected enabled 4g wireless world. 2003.

2. ITU-T Rec. P.800. Methods of Subjective Determination of Transmission Quality. ITU, 1996.

3. ITU-T Rec. G.107. The E-model, a Computational Model for Use in Transmission Planning. ITU, 2009.

4. ITU-T Rec. P.862. Perceptual Evaluation of Speech Quality(PESQ): An Objective Method for End-toEnd Speech Quality Assessment of Narrow-Band Telephone Networks and Speech Codecs. ITU, 2001.

5. Alexander Raake. Speech Quality of VoIP: Assessment and Prediction. John Wiley \& Sons, September 2006.

6. S. Möller, A. Raake, N. Kitawaki, A. Takahashi, and M. Wältermann. Impairment Factor Framework for Wideband Speech Codecs. IEEE Transactions on Audio, Speech, and Language Processing, 14(6):1969-1976, 2006.

7. N. Côté, S. Möller, V. Gautier-Turbin, and A. Raake. Analysis of a Quality Prediction Model for Wideband Speech Quality, the WB-PESQ. In Proceeding of the 2nd ISCA/DEGA Tutorial and Research Workshop on Perceptual Quality of Systems, pages 115-122, 2006.

8. S. Moller, M. Waltermann, B. Lewcio, N. Kirschnick, and P. Vidales. Speech quality while roaming in next generation networks, 2009.

9. M.A. Mehmood, B. Lewcio, P. Vidales, A. Feldmann, and S. Moeller. Understanding signal-based speech quality prediction in future mobile communications, 2010.

10. Blazej Lewcio, Marcel Wältermann, Sebastian Möller, and Pablo Vidales. E-model supported switching between narrowband and wideband speech quality. In Proceedings of First International Workshop on Quality of Multimedia Experience (QoMEX'09), San Diego, CA, United States, 2009.

11. K.T. Chen, C.Y. Huang, P. Huang, and C.L. Lei. Quantifying Skype user satisfaction. ACM SIGCOMM Computer Communication Review, 36(4):399-410, 2006.

12. R. L. Winkler. Decision modeling and rational choice: Ahp and utility theory. Manage. Sci., 36(3):247248, 1990.

13. Olga Ormond and John Murphy. Utility-based intelligent network selection. In in Beyond $3 G$ Systems, IEEE International Conference on Communications, 2006.

14. Rajkumar Buyya, David Abramson, Jonathan Giddy, and Heinz Stockinger. Economic models for resource management and scheduling in grid computing. pages 1507-1542. Wiley Press, 2002.

15. J.; Fang Zhu; McNair. Vertical handoffs in fourth-generation multinetwork environments. 2004.

16. Von Neumann. Theory of games and economic behavior. Manage. Sci., 1954.

17. C. Perkins. IP Mobility Support for IPv4. RFC 3344 (Proposed Standard), August 2002. Updated by RFC 4721.

18. Benny Prijono. Open Source SIP Stack and Media Stack for Presence, Instant Messaging, and Multimedia Communication. http://pjsip.org/.

19. Marcel Wältermann, Blazej Lewcio, Pablo Vidales, and Sebastian Möller. A Technique for Seamless VoIP-Codec Switching in Next Generation Networks. In Proceedings of IEEE International Conference on Communications (ICC 2008).

20. Pablo Vidales, Niklas Kirschnick, Frank Steuer, Blazej Lewcio, Marcel Wältermann, and Sebastian Möller. Mobisense Testbed: Merging User Perception and Network Performance. In Proceedings of the 4th International Conference on (TRIDENTCOM), 2008. 